Cahiers Société

\title{
Imaginaire instituant versus logique des sciences sociales
}

\section{Marc Maesschalck}

Numéro 1, mai 2019

Castoriadis et les sciences sociales

URI : https://id.erudit.org/iderudit/1068419ar

DOI : https://doi.org/10.7202/1068419ar

Aller au sommaire du numéro

Éditeur(s)

Collectif Société

ISSN

2562-5373 (imprimé)

2562-5381 (numérique)

Découvrir la revue

Citer cet article

Maesschalck, M. (2019). Imaginaire instituant versus logique des sciences sociales. Cahiers Société, (1), 17-33. https://doi.org/10.7202/1068419ar

\section{Résumé de l'article}

Alors qu'Habermas choisit de prendre ses distances par rapport aux ressources de la critique psychanalytique du malaise social, Castoriadis persiste dans l'intérêt qu'il porte à cette critique. À la différence d'Habermas, Castoriadis estime en effet qu'une critique de la raison prétendant faire la vérité sur la praxis reste incomplète si elle manque la tendance des représentations théoriques à supposer comme déjà donnée la coupure imaginaire dont elles procèdent. La prétendue « logique des sciences sociales » repose sur le déni du rapport inconscient au signifiant qui constitue le réel. 


\title{
Imaginaire instituant versus logique des sciences sociales
}

\author{
Marc MAESSCHALCK \\ Université catholique de Louvain
}

Lorsqu'on analyse l'épistémologie des sciences sociales implicite (Castoriadis) ou explicite (Habermas) de deux auteurs comme Castoriadis ou Habermas, un double mouvement saute aux yeux qui mérite, à notre sens, d'être clarifié. Là où Castoriadis maintient un rôle incontournable de l'approche psychanalytique pour interpréter l'illusion produite par un discours s'autonomisant toujours plus de son contexte de genèse, Habermas prend ses distances par rapport aux modèles interprétatifs hérités de Marcuse et Adorno pour renouer avec une approche positive de la normativité du discours. Au-delà d'un manque primitif ou d'une distorsion originaire hors d'atteinte, Habermas pense ainsi restaurer la capacité authentique du savoir à admettre sa propre condition d'opération toujours idéalisable. Cette idée d'une normativité de la raison soustraite imaginairement au travail du temps pose problème à Castoriadis, car elle reste indexée au mythe moderne d'une «définitisation» de la raison qui finit par considérer toute limite socialement identifiée comme un nouveau terrain d'exploration.

\section{Le contexte d'une science de la praxis}

À l'époque de la revue Socialisme ou barbarie et après les expériences décevantes tant du parti communiste grec que du trotskisme de la $\mathrm{IV}^{\mathrm{e}}$ Internationale à Paris ${ }^{1}$, Castoriadis s'est inscrit avec Claude Lefort dans une action intellectuelle forte contre les illusions d'un gauchisme dogmatique qui paraissait certain d'avance de sa vérité. Contre ce dogmatisme, force est de reconnaître que dans la pratique, la recherche d'une orientation révolutionnaire procède d'un débat constant et d'une réflexion critique non seulement sur les événements, mais surtout sur la manière de s'organiser pour y faire face. Il n'est pas seulement question de confronter la bureaucratie socialiste en URSS, mais aussi de remettre en question les appareils de parti, leur division

${ }^{1} C f$. Hans Joas, «L'institutionnalisation comme processus créateur. Sur la signification sociologique de la philosophie politique », dans Autonomie et autotransformation de la société. La philosophie militante de Cornelius Castoriadis, dir. Giovanni Busino, Genève, Droz, 1989, p. 174. Nous remercions pour son aide le chercheur au doctorat Oscar Palacios Bustamante (CPDR) avec qui nous publions une étude qui reprend et développe plusieurs des points abordés dans le texte qui suit. 
Imaginaire instituant versus logique des sciences sociales | Marc MAESSCHALCK

des tâches entre l'élite dirigeante et la base, les conflits idéologiques réservés aux intellectuels éclairés et les mots d'ordre destinés aux mouvements de masse.

C'est dans ce contexte critique que vont se forger les idées de social-historique ou d'imaginaire instituant. Il est possible qu'un rapport au signifiant maître échappe à ceux qui sont considérés uniquement comme les sujets désirant le changement social. Ont-ils même une histoire ? C'est une des questions lancinantes de Socialisme ou barbarie. Comment rendre aux concernés l'histoire du mouvement qu'ils sont censés incarner autrement que comme une théorie générale de la lutte des classes occupées à garantir l'avènement de l'ordre révolutionnaire? La seule manière d'y arriver est de procéder à partir de l'expérience du mouvement comme mode d'organisation. L'élément historique d'un mouvement c'est son évolution interne comme organisation analysée à partir de ceux qui s'y impliquent. L'organisation est un magma de décisions et de micropratiques qui distribue le pouvoir et irradie l'influence dans l'ensemble de la société. Contre une division essentialiste entre la classe et le parti, le mouvement qui travaille la conscience de classe en direction de l'action révolutionnaire ne joue que sur des distinctions provisoires : c'est le principe de l'entrisme dans sa version noble ; participer à égalité avec les autres acteurs concernés par des résistances sociales, s'activer avec eux pour affermir leur cohésion d'action, sans détourner pour autant le contrôle de celle-ci en amenant des idées produites hors du contexte d'action. La fonction pratique d'une réflexion révolutionnaire dans un milieu d'action n'est pas d'anticiper le cheminement de la conscience collective. Ce dernier dépend de la propre capacité à répondre aux situations d'action. Il est au contraire impératif de respecter ce rythme d'apprentissage, car il garantit la solidité des étapes réalisées par la conscience collective pour envisager des stratégies et des solutions adaptées. S'il est utile de permettre à des minorités critiques de se structurer dans le processus d'organisation porté par le commun des travailleurs en lutte, c'est uniquement pour soutenir la prise de conscience par cette organisation de la nécessité d'agir sur un plan global, donc de parvenir à se projeter au-delà des problèmes locaux sur le plan programmatique de l'institution sociale.

Il semble dès cette époque impossible qu'une organisation même progressiste et orientée vers le changement des rapports d'exploitation dans la société parvienne à se transformer sans se figer si ce n'est grâce à une «politisation accrue ${ }^{2}$ » de ses fonctions, en dépassant les partages de rôle entre intellectuels, élites dirigeantes, comités de lutte, sections de terrain. Tous ces clivages provisoires n'ont d'intérêt que s'ils agissent sur la conscience en formation pour qu'elle se saisisse, dans sa tendance à la lutte, comme une «tendance à l'autonomies ». Elle parviendrait de cette manière à

\footnotetext{
2 Note précédant le texte sur «Le parti révolutionnaire» dans Socialisme ou Barbarie, n 2, 1949, reproduit dans Cornelius Castoriadis, L'expérience du mouvement ouvrier, I. Comment lutter, Paris, Union Générale d'Édition, 1974, p. 170.

3 Ibid., p. 171.
} 
Imaginaire instituant versus logique des sciences sociales | Marc MAESSCHALCK

s'imaginer au-delà de ses clivages comme une nouvelle communauté d'action capable d'être sa propre avant-garde, avec « un programme politique complet et une conception historique des problèmes $4 »$.

De multiples éléments convergent dans cette vision structurale de la lutte sociale médiée par la prise de conscience de l'illusion bureaucratique. L'histoire du mouvement se noue avec une sociologie des organisations révolutionnaires et une anthropologie de l'action militante. L'accès immédiat aux effets de conscience par l'expérience de la lutte et les contacts avec elle permet d'établir un discours engagé qui ne cherche pas à s'imposer comme vérité, encore moins comme doctrine, mais à clarifier les choix pratiques à poser pour assurer les orientations révolutionnaires. C'est le pouvoir instituant du réel social-historique qui met en branle les débats, l'imminence des actions dans les effets d'exploitation et de résistance.

Il n'est pas toujours simple de se resituer dans ce contexte propre au discours général d'une science de la praxis qui sait déjà une histoire en train de se faire au prorata des rapports de production et des formes d'organisation imposées par la bureaucratie capitaliste. Le jeu économique est d'emblée prédéfini dans une fonction d'organisation de la production et la société sédimentée par ce partage productiviste ne répond à la taille des individus qu'en s'aliénant à ce processus ou en s'alliant pour se soustraire à l'enrôlement général. On n'est pas encore dans cette époque où, à côté des processus d'enrôlement dans la production, les superstructures sociales se sont constituées en nouvelles sphères de pouvoir : celle des médias et de la communication qui garantit la légitimité, mais aussi celle de la science comme puissance universelle de salut qui garantit la sécurité et le bien-être. Une science de la praxis attachée à la sphère de la production ne peut plus ambitionner le rôle général auquel elle prétendait encore naïvement dans les années 19605. Dans ce contexte, il était même possible de décliner les sciences selon leur option première par rapport à cette théorie générale de la praxis : économie marxiste, sociologie marxiste, anthropologie marxiste, et pourquoi pas, linguistique marxiste et psychanalyse marxiste... L'illusion historique qui prévalait à la croyance dans la possibilité d'une telle division a eu des conséquences au-delà de ce que dénonce une épistémologie bien-pensante. On aurait de fait pu considérer de manière minimaliste qu'il s'agissait tout simplement d'hypothèses de base, au même titre que l'empirisme ou le positivisme qui dérangent moins. Sont-elles impossibles à falsifier ? Castoriadis a toujours exprimé des réserves à l'égard des critiques falsificationnistes ${ }^{6}$ : pour falsifier, il faut toujours présupposer

\footnotetext{
4 Idem.

5 Cf. Gérald Berthoud, « Castoriadis et la critique des sciences sociales », dans Autonomie et autotransformation de la société, op. cit., p. 444.

6 Cornelius Castoriadis, "Imagination, imaginaire, réflexion », dans Les carrefours du labyrinthe, $V$. Fait et à faire, Paris, Seuil, 1997, p. 279-280 ; on verra aussi Cornelius Castoriadis, "Portée ontologique de l'histoire de la science ", dans Les carrefours du labyrinthe, II. Domaines de l'homme, Paris, Seuil, 1986, p. 452.
} 
un quelque chose à falsifier, qui ne se réduit donc pas à un «falsifiable», sans quoi il ne serait qu'un préconstruit qui satisfait les critères de l'opération supposée le tester... La véritable falsification dans les productions scientifiques provient d'un déplacement de point de vue qui permet d'interroger suivant de nouveaux critères les objets déjà connus. La psychologie rationnelle a exploré les limites de la conscience et identifié ses propres limites, tels les paralogismes de Kant. Mais une théorisation de l'inconscient qui détruit l'holisme de la représentation et établit une relation de perturbation entre les traumatismes refoulés et la vie consciente ouvre de nouvelles pistes d'investigation. Des affirmations antérieures sur la maladie mentale peuvent être falsifiées, l'influence du passé revisitée, etc. Ces déplacements conduisent à de nouvelles «figures imaginaires d'intelligibilité7 ». Le problème d'un discours générique sur la praxis ne se situe pas sur ce plan. On pourrait également reprendre la manière dont Althusser interprète l'écart ou le changement de terrain ${ }^{8}$ réalisé par Marx vis-à-vis de la notion de travail chez Smith ou Ricardo. Par contre, la dislocation des privilèges modernes de la sphère productiviste/industrielle a d'autres conséquences sur le plan du rapport aux sciences sociales. On ne peut simplement considérer celles-ci comme un discours super-structurel voilant les pratiques répressives de l'infrastructure productiviste : il y a des sciences bourgeoises, des sciences impérialistes/coloniales, des sciences au service de l'appareil bureaucratique, etc. Mais qu'en est-il lorsque ce rapport présupposé est renversé, quand ce n'est plus l'appareil bureaucratique qui met la science à son service, mais la science qui s'autonomise et façonne l'existence bureaucratique par l'exercice d'un pouvoir recalibré par elle sous la forme d'une administration du vivant?

\section{L'autonomisation des sciences sociales comme pratique discursive}

Une science qui a la capacité d'induire les modes de transformation de l'être socialhistorique en déterminant l'ordre des questions recevables et urgentes, de même qu'une sphère de légitimité communicationnelle qui autorise les significations axiologiques acceptables et ceux qui les représentent - ces entités fantasmatiques d'un nouveau genre ne sont plus incluses dans l'horizon de jouissance de l'univers productiviste. Même du point de vue de la critique que nous avons rappelé brièvement, elles appellent des moyens d'approche différents : le contact immédiat avec une conscience en formation dans des processus de résistance localisés ou ancrés n'a plus de sens. L'organisation révolutionnaire d'avant-garde n'a plus de corps social spécifique sur lequel greffer son rythme pour interagir dans le sens d'une politisation accrue. Le point de vue des sciences sociales, ou tout au moins, leurs « figures nouvel-

\footnotetext{
$7 \mathrm{Cf}$. Cornelius Castoriadis, «Imagination, imaginaire, réflexion », dans Les carrefours du labyrinthe, $V$, op. cit., p. 280.

${ }^{8}$ Louis Althusser et al., Lire le Capital, Paris, Maspero, 1970, vol. I, p. 28.
} 
Imaginaire instituant versus logique des sciences sociales | Marc MAESSCHALCK

les d'intelligibilité » peuvent ouvrir de nouveaux « effort[s] pour briser la clôture où nous sommes chaque fois nécessairement pris comme sujets 9 ».

L'évidence de la praxis comme problème général a écarté l'intérêt pour un déplacement inhérent aux approches scientifiques des questions sociales. Il ne s'agit pas uniquement des déplacements paradigmatiques comme ceux impulsés par la linguistique structurale ou par la pragmatique communicationnelle. Ce qui importe également ce sont les écarts inhérents à ces pratiques elles-mêmes qui ont amené les sciences sociales à traiter sur de nouvelles bases les questions d'intersubjectivité (comme dans l'interactionnisme), les questions de cognition (comme dans le computationnalisme), voire les questions de genre et de transhumanisme. En devenant productrice de l'ordre social, les sciences ont aussi tenté de modifier le sens du politique et la construction de la responsabilité collective. Elles ont tenté de revoir les théories de l'action et de la norme qui les sous-tendent pour formuler de nouveaux modèles coopératifs basés par exemple sur le format des réseaux.

Il y a donc au-delà des expériences qui ont pu être liées à une théorie de la praxis et à l'exploration des modes de conscientisation articulés aux résistances sociales, des formes d'autotransformation des sciences qui ne se résument pas à des modifications de discours, mais qui correspondent concrètement à des modifications de praxis dans la production de l'être social-historique. Ce que Castoriadis observait au niveau des groupuscules militants, délibérant entre eux pour ensuite innerver les pratiques autour d'eux, se produit aujourd'hui dans des formes d'organisation de la recherche et de l'innovation pour produire des collectifs intelligents susceptibles de construire de nouvelles solutions. Dans ces contextes liés à la gestion organisationnelle, il ne fait aucun doute que le problème envisagé par Castoriadis, le recours à l'éthique organisationnelle, se retrouve, au sens où la question des souffrances engendrées par la manipulation des ressources affectives des individus ne peut se résoudre que par une politisation accrue de leurs pratiques, c'est-à-dire par la mise en question d'une vision plus complète du programme auquel ils participent et par la reconnaissance de leur capacité d'autonomie à l'intérieur de celui-ci.

Pour évoluer vers ces questions, les sciences sociales ont parcouru différents chemins qu'une philosophie contemporaine de l'être social-historique ne peut ignorer. Mais un tel rapport aux sciences sociales et à leurs évolutions doit se construire notamment en ouvrant des «brèches dans la clôture où la pensée tend toujours à s'enfermer de nouveau ${ }^{10} »$.

\footnotetext{
9 Cornelius Castoriadis, «Imagination, imaginaire, réflexion », dans Les carrefours du labyrinthe, V, op. cit., p. 280.

${ }_{10}$ Idem.
} 


\section{La logique des sciences sociales}

La façon dont procède Habermas consiste à tenter de mieux comprendre comment les sciences sociales ont modifié leur rapport aux phénomènes et aux interprétations, à la fois en abandonnant la théorie kantienne du transcendantal et évitant le recours à des totalités culturelles fermées sur elles-mêmes. Le besoin de théorisation des sciences sociales est donc considéré en rapport à une question qui échappe à la simple observation et au calcul, celle de la production d'orientations pratiques pour compenser l'absence toujours plus évidente de substrats naturels pour la maîtrise technique de l'existence ${ }^{11}$. Il s'agit d'action anticipative, mais sans pouvoir se limiter pour autant à une forme de normativisme ni à une forme de fonctionnalisme. En fait, l'activité sociale n'est productive que parce qu'elle combine deux niveaux, celui, intentionnel, des interactions significatives dans la communication et celui, involontaire, des comportements compulsifs qui s'attachent à la répétition ${ }^{12}$. C'est en observant cette évolution des sciences sociales qu'Habermas cerne le rôle qu'y jouera désormais l'activité communicationnelle pour dépasser les seuls effets de canalisation des comportements par le système technico-administratif et restaurer les effets de sens qui visent l'interruption de la domination. Dans ce cas, le langage n'est plus simplement une manière de décrire le monde ou de l'énoncer comme référence, mais aussi une façon de créer du sens partagé en s'emparant de ces relations au monde pour les soumettre à l'assentiment d'autrui ${ }^{13}$. La théorisation de l'interaction discursive devient capitale pour toute science de l'activité sociale.

En formulant ces recommandations normatives ${ }^{14}$, Habermas bascule lui-même du descriptif au pragmatique: il renforce une orientation encore indécise et implicite vers une pragmatique universelle. L'important, nous semble-t-il, dans son rapport aux sciences sociales est moins dans cette interaction qu'il tente de créer que dans le point de levier qui lui permet d'y arriver, à savoir la question de l'action et celle, conséquente, d'un concept d'action suffisamment fécond pour les sciences sociales ${ }^{15}$, c'est-à-dire qui ne se contente pas d'endosser le clivage traditionnel entre des structures d'arrière-plan guidant l'adoption de rôles et des ressources réflexives permettant de construire des consensus interprétatifs sur les valeurs. Habermas suggère que c'est l'entre-deux langagier rendant possible le couplage de ces deux dimensions qui doit

\footnotetext{
11 Jürgen Habermas, Logique des sciences sociales et autres essais, Paris, Presses universitaires de France, 1987, p. 112.

12 Ibid., p. 116.

13 Ibid., p. 429.

14 On lit (idem) : «Le concept d'activité communicationnelle entraîne l'obligation de considérer les acteurs comme des locuteurs et des auditeurs qui se réfèrent à quelque chose appartenant au monde objectif, social ou subjectif, tout en émettant, les uns vis-à-vis des autres, des prétentions à la validité susceptibles d'être acceptées ou contestées ».

15 Ibid., p. 424.
} 
être élucidé pour lui-même comme forme décentrée d'imbrication du monde social et du monde vécu' ${ }^{16}$.

Rainer Rochlitz, le traducteur de Logique des sciences sociales, considère que la démarche d'Habermas adopte une posture thérapeutique semblable à celle de la psychanalyse ${ }^{17}$. Le théoricien accompagne une forme d'auto-analyse des méthodologies pour reconstruire la forme matricielle dont le manque ne cesse de se répéter dans les multiples versions de la médiation linguistique mobilisée par les théories de l'action sociale. Les visées de performances, de résultats, d'efficacité, ne cessent de bloquer le retour d'une communication libérée de ces contraintes de succès social. Dans ce cas, la référence à Alfred Lorenzer devrait s'avérer décisive ${ }^{18}$.

Pourtant, cette lecture doit être remise en question. C'est précisément lorsqu'il traite des thèses de l'herméneutique des profondeurs qu'Habermas prend ses distances avec les schémas explicatifs qu'elle mobilise pour considérer, en fonction de ses catégories fondamentales, l'expérience naturelle du langage comme dominée par une perte ou un processus de désymbolisation. L'accès à une forme potentielle de la communication intersubjective est ainsi barré au profit d'un savoir d'expert qui décode les manœuvres des communicants, prisonniers du processus de déformation de leurs visées expressives. Habermas pense au contraire qu'il faut éviter le recours prématuré à un tel appareil critique pour comprendre ce qui est en jeu dans l'échange intersubjectifí. La psychanalyse appliquée au champ des interactions sociales est donc chargée de présupposés sur l'origine du langage qui oblitèrent sa fonction première d'imbrication des mondes dans l'unité du réel.

Dès cette époque (1970), Habermas prend donc ses distances avec le modèle thérapeutique qui a dominé l'École de Francfort. Si l'on considère la société dans son ensemble comme le symptôme d'une pathologie productiviste déterminée par les modes d'organisation du capitalisme avancé, il est impossible de renouer avec une compréhension non déformée de l'activité communicationnelle ${ }^{20}$. Pourtant, l'enjeu de toute compréhension réside bien dans la capacité de renouer avec une scène primitive où les compétences communicationnelles peuvent être détachées de la genèse de leur déformation et être appréhendées comme une forme potentielle ou une matrice d'interaction sociale à même de guider vers des processus d'entente sans contrainte ${ }^{21}$. Ce qu'indique Habermas de cette manière, ce n'est pas uniquement le futur pro-

${ }^{16}$ Ibid., p. 446.

17 Rainer Rochlitz, « Avant-propos du traducteur », dans Logique des sciences sociales, op. cit., p. xii.

18 Jürgen Habermas, Logique des sciences sociales, op. cit., p. 254 sq.

19 On lit, par exemple, dans les Christian Gauss Lectures : « en accomplissant directement une interaction, nous sommes incapables d'imputer à un vis-à-vis que nous rencontrons comme un autre Je, des motifs inconscients ». Jürgen Habermas, Sociologie et théorie du langage, Paris, Armand Colin, 1995, p. 120.

20 Jürgen Habermas, Logique des sciences sociales, op. cit., p. 255.

21 Ibid., p. 267. 
gramme de recherche sur l'activité communicationnelle, mais surtout l'impossibilité pour l'usage du langage dans le schéma thérapeutique de la psychanalyse de jouer ce rôle de guide. Un tel rôle ne peut être reconnu à l'activité langagière que si elle est reconstruite du point de vue de sa propre précompréhension comme forme discursive de l'intercompréhension. Il y a préalablement, pour toute science de l'action, une idée régulatrice de l'activité linguistique comme processus de convergence des points de vue dans une communauté capable de garantir de manière illimitée et hors de toute contrainte la formation de consensus ${ }^{22}$.

Chez Habermas, c'est bien le dialogue avec les sciences sociales du point de vue de leurs présuppositions qui conduit à la reformulation d'une pragmatique universelle. Toutefois, la question de l'action basée sur des conditions minimales d'intercompréhension n'est pas la seule à avoir joué un rôle décisif dans la transformation des sciences sociales au $\mathrm{XX}^{\mathrm{e}}$ siècle. Habermas opte pour une méthode d'idéalisation des présuppositions en vue de cerner un horizon régulateur de l'action langagière. Il participe encore en ce sens de ce que Putnam nomme un holisme sémantique ${ }^{23}$. On pourrait procéder différemment en revendiquant notamment un holisme pragmatique, lequel n'a pas besoin en soi d'idéalisation des présuppositions, mais uniquement de croyance dans des processus d'approximation ou de levée partielle de l'indétermination, de façon à déplacer et à redéfinir indéfiniment les désaccords ou les dissensus. Cette idée se retrouve notamment chez James Bohman et chez Charles Taylor. Mais elle a surtout permis un autre usage de la pragmatique au sens plus traditionnel des pragmatistes nord-américains comme un processus d'enquête permettant de revoir des croyances dans l'expérimentation de solutions à des problèmes ${ }^{24}$. La théorie des organisations a été profondément marquée par cette vision, de même que la théorie des normes juridiques25. On trouve aussi chez un praticien comme Stephen Mitchell une révision de la théorie du dialogue psychanalytique à partir de cette compréhension plus expérimentale de l'interaction langagière ${ }^{26}$. On touche ainsi de manière plus générale à ce qu'il est convenu d'appeler aujourd'hui le tournant pragmatique des sciences sociales ${ }^{27}$ qu'Habermas n'a que très partiellement travaillé et qui montre l'intérêt de la poursuite de ce genre de dialogue. Procéder comme Habermas permet de fait d'identifier des présuppositions et donc de reconstruire des décalages qui amènent à sortir de l'isolement des théories trop générales de l'être social historique, comme celles du marxisme ou du structuralisme.

\footnotetext{
22 Ibid., p. 269.

23 Hilary Putnam, Le réalisme à visage humain, Paris, Seuil, 1994, p. 493-494.

24 Charles F. Sabel, Learning by Monitoring, Cambridge, Harvard University Press, 2006.

25 Jacques Lenoble et Marc Maesschalck, Toward a theory of governance : the action of norms, The Hague/London/New York, Kluwer Law International, 2003.

26 Stephen A. Mitchell, Relationality. From attachment to intersubjectivity, Hillsdale, Analytic Press, 2000.

27 Alfonso Morales (dir.), Renascent pragmatism, Aldershot, Ashgate, 2003.
} 
Imaginaire instituant versus logique des sciences sociales | Marc MAESSCHALCK

\section{L'inconscient des sciences sociales}

Cette relation d'ordre thérapeutique avec les sciences sociales qui se base sur l'identification et l'analyse des présuppositions théoriques n'existe pas chez. Étonnamment, chez Habermas, elle conduit à son abandon au nom même de ce travail sur les présuppositions. De fait, la déformation toujours déjà supposée de l'activité langagière dans les pratiques sociales bloque l'accès à l'idéalisation d'un modèle capable d'orienter l'activité thérapeutique. Il y a dans la démarche interprétative des déformations subies par l'action rationnelle et ses justifications une sorte de forçage de l'interprétation qui conduit à la répétition du manque interrogé : il y a toujours contrainte de la communication dans un monde soumis à l'activité instrumentale et à la poursuite des intérêts. Il faut donc suspendre le schéma analytique pour interrompre la répétition et éviter de postuler que tout idéal de communication sans contrainte dépend fondamentalement de la reconnaissance pratique de « la non-vérité effective et de la violence qui se perpétue ${ }^{28} \gg$ dans nos formes réelles d'intercompréhension. Habermas pense ainsi atteindre une limite de la démarche analytique qui contraint celle-ci à accorder une autorité à la violence légitimée dans l'ordre de la raison sociale 29 .

Chez Castoriadis, ce point de non-retour n'a pas de sens. Le rapport entre l'action analytique et la société reste décisif. Certes chez Habermas, à l'encontre du postulat sur le manque qui continue de cliver nos pratiques d'intercompréhension, une pragmatique universelle tente une réduction de ce clivage en prenant appui sur le rapport au réel qui se joue dans le discours, à savoir non l'établissement d'une vérité imaginaire de la pratique au-delà des violences qui la déforment, mais l'instauration d'une forme de «vie vraie » conforme au désir de partage d'un monde commun. Cependant, le présupposé qui a conduit le travail d'Habermas réside dans une prise en compte du travail de théorisation des sciences sociales s'efforçant de dépasser, par leur concept d'action, l'indétermination entre intention signifiante et contrainte de reproduction dont elles pensent procéder. Mais ce qui demeure problématique dans cette démarche et conduit à des constructions de plus en plus absconses pour déconstruire ce rapport à l'indétermination c'est l'ordre représentationnel de la vérité ainsi convoqué et admis comme point de départ. Si pour Castoriadis, la démarche analytique reste primordiale dans une approche critique des transformations sociales, c'est parce que, selon les termes d'Habermas, l'opposition entre le factuel et l'idéal dérive inévitablement d'une "fausse conscience" de la réalitéso. Dans le rapport à la transformation des formes de vie, la référence au jeu des représentations doit ellemême être mise en question de manière à « éprouver les limites de l'arbitraire de

\footnotetext{
28 Jürgen Habermas, Logique des sciences sociales, op. cit., p. 268. Habermas cite Albrecht Wellmer, Kritische Gesellschaftstheorie und Positivismus, Frankfurt am Main, Suhrkamp, 1969, p. 48.

29 Ibid., p. 272.

30 Ibid., p. 270.
} 
l'institution social historique ${ }^{31} »$. L'ordre représentationnel qu'explore Habermas inscrit l'analyse dans le temps institué du repérage imaginaire, le temps du "représenter social $3^{2}$ ». Il répète de cette manière la négation inhérente à l'être social-historique, à savoir le déni du faire, de l'autocréation chaotique de l'identité à soi, le kairos d'un surgissement «non déterminabl[e] d'avance33», une sorte de rupture dans la continuité, une altération du même. Pour Castoriadis, c'est ce point de suspension dans la croyance en l'identité à soi qu'il faut éviter d'oblitérer, car c'est par là que chaque forme de vie est projetée dans le temps, qu'elle laisse son rapport chaotique au signifiant pour s'inscrire dans le flux des signifiés.

La question est effectivement celle du flux, non pas celle du flux institué dans sa forme et sa continuité, mais celle de l'entrée dans le flux comme perpétuel devenir, comme occasion toujours relancée, pouvoir de bifurcation et de rupture34. Ce que le travail psychanalytique met en jeu c'est une certaine capacité de lucidité par rapport à l'origine des normes et des institutions, l'absence de cause toute puissante échappant à notre pouvoir de création, la contingence qui est au principe de l'autonomie. Il y a création au sens fort, sans dérivation ni explication causale, par interruption de la répétition et donc contre toute représentation prédonnée d'une omnipotence des rôles constitutifs, une fois pour toutes, de la structuration des formes de vie. L'intérêt de la psychanalyse réside dans le travail qu'elle tente de réaliser sur les attaches inconscientes des individus. Leur besoin de refuges, de prétextes devant l'inconnu ou l'incertain, leur attrait pour la force ou la violence35, l'abandon de l'initiative pour des garanties de vérité ou de sécurité36. Ce sont des illusions de notre imaginaire qui cherche à satisfaire le fantasme primaire de satisfaction totale des désirs. Ce fantasme nous inscrit dans un schéma pulsionnel binaire où soit le soi soit l'autre devient l'objet total du désir, alors que la vie sociale ne parvient à se réaliser que dans un mouvement de « tercéisation » : accepter l'autolimitation des désirs en passant par la négociation du rapport aux flux des signifiés, créer des rôles, introduire de l'indétermination. Le travail psychanalytique se confronte aux résistances les plus fondamentales de notre désir à se saisir de son autonomie pour oser l'espace incertain des relations d'égalité : le renoncement à l'omnipotence du père ou de la mèrepasse par un serment entre frères et sœurs 37 pour qu'un avenir puisse être produit de manière autonome, c'est-à-dire puisse mettre en question les lois mêmes de son existence38.

\footnotetext{
${ }^{31}$ Cornelius Castoriadis, L'institution imaginaire de la société, Paris, Seuil, 1999, p. 303.

32 Ibid., p. 314.

33 Ibid., p. 316.

34 Ibid., p. 319.

35 Cornelius Castoriadis, "Psychanalyse et société, I », dans Les carrefours du labyrinthe, II. Domaines de l'homme, op. cit., p. 40.

36 Ibid., p. 36.

37 Ibid., p. 46.

38 Cornelius Castoriadis, "L'imaginaire : la création dans le domaine social-historique ", dans Les carrefours du labyrinthe, II. Domaines de l'homme, op. cit., p. 237.
} 
Imaginaire instituant versus logique des sciences sociales | Marc MAESSCHALCK

Les sciences sociales produisent leurs analyses à l'inverse de ce schéma d'émancipation. Elles visent des régularités, des explications pour se saisir des conditions par lesquelles le changement parvient à se représenter comme une forme possible, acceptable, de détermination. C'est ce qui préoccupe le jeune Jakobson quand il s'en prend aux théories classiques du langage. Ce qui leur fait défaut c'est une appréhension du moment de déstructuration de leurs ensembles, le moment d'effacement des limites par lesquelles un ordre normatif institue le signifié. La déstructuration repousse le dire de l'énonciation à l'origine de son pouvoir: celui d'organiser des relations, de distinguer des mots, des choses, et des actes de locution qui se saisissent du rapport au signifiant pour l'assigner, l'enclore. Il faut ce quid pro quo originaire et permanent dans les entrelacs du langage (ses relations signitives 39 ) pour que des quasi-équivalences puissent se fixer dans un système d'usages, donner au poème de la langue sa forme instituée 40 .

\section{Conséquences du déni de l'inconscient}

Le déni de ce préalable déstructurant non seulement à la source de toutes les déterminités instituées, mais aussi inhérent à elles, est au principe des représentations de la logique des ordres institués par la montée en puissance de la raison calculatrice : la mathématique économique, le probabilisme social, sont autant de variantes de ce déni qui n'a cessé de gagner du terrain en sciences sociales, même dans le concept politique d'État bureaucratique ${ }^{41}$. La force de ce mouvement est d'être parvenu à absorber sa propre négation de l'indéterminé sous la forme d'une condition nécessaire à son prolongement. L'absorption de l'indétermination consiste à y voir un principe de développement plus radical que celui d'une idée régulatrice pour Kant ou d'une forme utopique du destin pour la Renaissance. Ce n'est pas l'absence de contenu déterminable de sa visée qui permet à la rationalité sociale de continuer à se projeter, pas même le quasi transcendantal d'une communauté idéale de communication sans

contrainte, c'est au contraire la possibilité d'user de ce contenu comme une limitation provisoire, une nouvelle situation limite à explorer pour déplacer le sens de la finnt2. C'est l'idée d'une forme de métadétermination pour laquelle seule la définitude a du

\footnotetext{
39 Ibid., p. 230.

40 En particulier, Roman Jakobson, « Notes marginales sur la prose du poète Pasternak » [1935], dans Huit questions de poétique, Paris, Seuil, 1977, p. 51-75. Une approche à comparer avec le beau texte d'Édouard Glissant, «L'intention poétique », dans L'intention poétique, Poétique II, Gallimard, Paris, 1997, p. 31-52.

${ }^{41}$ Cornelius Castoriadis, " Réflexions sur le "développement" et la "rationalité" ", dans Les carrefours du laybrinthe, II. Domaines de l'homme, op. cit., p. 140-141.

42 Un point qu'Agamben a particulièrement bien saisi dans l'usage technique de la situation-limite dans les camps de détention (Gorgio Agamben, Homo Sacer I, Le pouvoir souverain et la vie nue, Paris, Seuil, 1995, p. 202).
} 
sens : la détermination à redéterminer, un monde sans animaux, un monde sans air respirable, un monde écologiquement détruit...

Ce à quoi ne parviennent pas à échapper les sciences sociales c'est à une croyance inconsciente qui dirige toutes leurs représentations, celle de la séparabilité totale du pouvoir de rationalisation de l'être social-historique avec l'autre qui lui échappe constamment, l'imprévisibilité de son existence. Il ne s'agit pas de réaffirmer de cette façon des hypothèses de rationalité limitée pour mettre en cause les croyances optimalistes de la rationalité parfaite ni de se rabattre sur des principes de sous-optimalité en valorisant les régimes non standards de développement. Les remarques de Castoriadis entraînent bien plus loin. C'est la capacité de la raison déjà instituée à se confirmer dans une forme de temporalité disjointe de ses conséquences historiques qui pose question. On peut sans difficulté imaginer que les résultats escomptés ne seront pas au rendez-vous et que les engagements sur le climat, par exemple, seront l'objet de toutes les spéculations dans un contexte d'asymétrie d'information. Chaque nouvelle situation sera néanmoins une occasion supplémentaire pour rethéoriser et modéliser les choix rationnels en régime d'incertitude. Or c'est cette temporalité disjointe de la représentation scientifique que Castoriadis veut mettre en cause. Elle nous aliène de notre autonomie parce que celle-ci dépend de la capacité à partir de la crise pour interrompre la répétition.

Pour Habermas, la raison finit par s'épuiser dans le discours négatif soutenant l'auto-analyse de ses limites. Dans les termes qu'Habermas connaît chez Marcuse, reprenant notamment à sa façon Schiller43, il y va encore, dans la raison négative, d'une ultime et fondamentale négation, la désublimation du rapport à la compétence esthétique, la négation de la négation du rapport répressif aux pulsions. Par cet ultime revirement face à la pression exercée tant par le réel que par ses manques, la raison sociale parviendrait à se désempêtrer de son pouvoir négatif limité au contrôle des appétits pulsionnels 44 pour reconnaître, dans le sens du jeu et de la transgression, sa propre condition primitive 45 , à savoir un principe de jouissance désublimée, libérée du trauma de la transformation répressive des pulsions ${ }^{46}$.

Mais plus que Marcuse, Adorno est celui qui a mené jusque dans ses derniers retranchements l'auto-interprétation de la négativité comme suspension définitive de toutes les ruses à l'égard du temps, de l'autre et de la vérité. En même temps, cette impuissance à interrompre le procès du négatif et à relancer une rationalité postcritique montre la faiblesse d'une perspective adossée jusqu'au bout à l'auto-analyse d'un malaise qui procède des illusions transcendantales de la subjectivité.

43 Herbert Marcuse, «La notion de progrès à la lumière de la psychanalyse ", dans Culture et société, Paris, Minuit, 1970, p. 368.

44 Herbert Marcuse, «Liberté et théorie des pulsions », dans Culture et société, op. cit., p. 342.

45 Jürgen Habermas, Le discours philosophique de la modernité, Paris, Gallimard, 1988, p. 57.

46 Herbert Marcuse, « Liberté et théorie des pulsions », dans Culture et société, op. cit., p. 351. 
Imaginaire instituant versus logique des sciences sociales | Marc MAESSCHALCK

L'objectivation de l'entre-deux de la communication, l'intersubjectivité comme forme d'une pragmatique universelle, relance l'idéal d'un ordre rationalisable par les effets de la communauté conversationnelle. L'activité communicationnelle fonctionne sur le modèle d'une action réciproque tentant constamment de soumettre l'ouverture au monde du langage au test permanent de sa validation intramondaine comme fondement d'une forme de vie en commun 47. C'est bien d'achèvement de la modernité dont il s'agit même si se maintient un rapport à l'incertitude de cet achèvement qui dépend d'un pouvoir à co-construire en permanence et à instituer dans la durée.

Contre les représentations issues de l'historicisme linguistique privilégiant exclusivement la puissance d'autoréalisation du langage, Habermas croit dans la justesse du tournant des conceptions du langage porté par le pragmatisme et le structuralisme génétique 48 : la forme autoproductive de la performativité langagière renvoie nécessairement au travail intrasocial de sa mise en œuvre comme épreuve d'un sens partagé. La "transcendance de l'intérieur » chez Habermas renvoie à cette capacité coopérative du langage qui destine les locuteurs ordinaires à composer avec autrui, à apprendre à créer des relations durables avec autrui pour affronter l'incertitude des situations.

Ici encore, un appui discret sur le tournant pragmatique des sciences sociales et le « changement de structure des conceptions du monde 49 » fait toute la différence avec Castoriadis et avec le rôle qu'il continuera toujours d'accorder à la psychanalyse. Pour Castoriadis, même cette confiance première dans la discursivité des savoirs et de l'analyse reste à interroger, précisément parce qu'elle suspend le rapport à l'indétermination originaire des représentations. L'arrachement à l'en-soi des formes social-historiques du discours, même pour tester l'a priori concret5o de l'ouverture au monde, n'est pas le dernier mot du travail sur l'origine du rapport au signifiant. La seule manière de se situer par rapport à l'indéterminable, c'est de l'éprouver comme temps spécifique ou événement, comme un kairos $^{51}$ dans son surgissement imprévisible, une forme d'interruption dans le flux de la répétition.

\section{Suspendre le désir de répétition}

L'illusion fondamentale à laquelle reconduit la psychanalyse réside dans cette espérance téléologique qui consiste à maintenir la continuité du représenter en considérant que la crise d'une forme de rationalisation ne fait qu'appeler sa relève par une

47 Jürgen Habermas, Le discours philosophique de la modernité, op. cit., p. 379.

48 Ibid., p. 378.

49 Idem.

50 Ibid., p. 380.

${ }^{51}$ Cornelius Castoriadis, L'institution imaginaire de la société, op. cit., p. 315. 
autre rationalité. Pourtant, la relance éclairée du flux des discursivités sociales pardelà les crises momentanées ne fait qu'occulter le biais inconscient qui dirige cet effort de relance. Ce qui est ainsi biaisé c'est le rapport avec la limitation : il ne s'agit pas simplement d'y saisir un principe d'autolimitation, encore moins un frein comme le serait celui d'une modernité devenue enfin réflexive. Le rapport à reconstruire est celui d'une dé-limitation, d'une forme de transitivité qui porte à expérimenter l'incertain, à l'utiliser pour envisager un autre type d'au-delà du présent. C'est cet usage - pourtant refoulé par la continuité représentationnelle - qui parvient à nous mettre en transmutation dans un temps déjà disjoint, le "temps du faire ». Tout le problème pour Castoriadis réside dans ce désir inconscient de maîtriser le pouvoir de transmuter pour gagner en surplomb sur le changement et ses incertitudes, pour circonscrire l'imaginaire instituant dans une téléologie secrète du progrès et de la surhumanisation.

Comment éviter dès lors le pressentiment d'Habermas pour qui l'autonomie dont parle Castoriadis manque fondamentalement sa co-construction intersubjective comme condition de réalisation? Elle ne serait au bout du compte que l'effet d'une croyance radicalisée dans l'idéalisation du pouvoir expressif de la raison ${ }^{2}$, au même titre que la structure pulsionnelle chez Freud et, avant lui, Schiller. D'un côté, cet imaginaire social qui échappe à chaque individu comme arrière-plan créateur est en même temps ce qui se récupère en lui subjectivement comme flux des signifiés53. D'un autre côté, rendre conscient par l'analyse ce socle primitif permanent, cet Urgrund, ce serait ipso facto le libérer de l'emprise des déterminations connues d'avance et le rendre à son autonomie54. Habermas est persuadé que le prix à payer, pour saisir, avec Castoriadis, la pleine puissance d'autonomie et donc d'interruption des répétitions de sens par cet imaginaire instituant, réside dans l'isolement ontologique de l'être du social-historique, disjoint de sa validation comme sens intramondain et donc comme mode d'apprentissage de ses propres règles de signification et d'autocompréhension 55 .

À notre sens, c'est la philosophie de l'événement de Badiou qui permet de lever ce malentendu. En optant formellement pour une disjonction, Habermas manque la question centrale de la relation entre le temps du faire et celui du représenter, entre celle du rapport subjectif entre l'un vécu et l'identique où il survient, mais aussi celle du rapport objectif entre l'événement qui advient dans un monde comme changement maximal d'apparaître et l'événement qui advient formellement comme ensemble appartenant à soi-même ${ }^{6}$. Si les mathématiques permettent de traiter la multiplicité

\footnotetext{
52 Jürgen Habermas, Le discours philosophique de la modernité, op. cit., p. 377.

53 Ibid., p. 376.

54 Ibid., p. 378.

55 Il s'agirait d'un savoir de l'effectif sans prise sur sa transformation réelle comme norme d'action.

$5^{6}$ Badiou reconnaît d'ailleurs lui-même qu'il n'est pas clair de déterminer si l'événement peut subsister singulièrement comme une différence immanente à la consistance du monde ou s'il est nécessaire que
} 
Imaginaire instituant versus logique des sciences sociales | Marc MAESSCHALCK

des occurrences sans recours à une unité totalisante, l'ontologie transcendantale des modes d'apparaître permet de son côté d'insérer les occurrences selon des degrés d'intensité dans un réseau d'apparaissance57. L'onto-logie mathématicienne permet de formaliser et comprendre tout ce qui est, apparait ou advient. Mais la question se pose encore de la relation entre cette ontologie formelle et les aspects topologiques du système qui sont nécessaires pour comprendre un monde en tant qu'apparaître de la multiplicité, en fonction d'un réseau transcendantal de degrés d'apparaître58.

C'est sur ce second plan que peut se poser le problème qui nous semble prolonger l'approche de Castoriadis, à savoir celui de la différence entre l'événement comme changement maximal d'existence ou d'apparaître et l'événement comme ensemble qui appartient à soi-même. Est-il possible de concevoir un rapport primordial avec les événements qui nous en rendrait les co-créateurs, mais sans pour autant qu'il s'agisse uniquement de s'identifier à eux, d'y être incorporé comme part du social ? La référence à l'imaginaire peut-elle parvenir à se stabiliser dans une opération de coconstitution sans se réduire à la simple répétition du même et donc sans annuler l'incorporation du signifiant dans le flux des signifiés ?

Premièrement, suivant un tel rapport primordial, le social pourrait trouver son équilibre ontologique sans s'adosser au cadre contraint des normes de coopération et de consensus, c'est-à-dire sans se maintenir conventionnellement comme structuré par des normes imposées de l'extérieur et imaginairement détachées de ses capacités subjectives, c'est-à-dire l'idéalisation d'une communauté de communication sans contrainte.

Deuxièmement, ce rapport d'équilibre co-institué renvoie le sujet à une différence immanente dans son rapport à l'identique : il n'est pas uniquement une variante de l'univers constitué par les fluctuations événementielles59. Dans ce cas, il pourrait être conçu comme entretenant un rapport primordial avec les événements qui le concernent, à tel point qu'il en serait à sa manière co-créateur, mais sans pour autant s'identifier à eux. Grâce à une telle approche topologique de la non-identité des sujets historiques, le social pourrait regagner les capacités de se gouverner intérieurement, en assumant son indétermination.

toute consistance de l'être soit toujours comprise à la manière d'une fluctuation entre un ou plusieurs événements (Alain Badiou, Logiques des mondes. L'être et l'événement 2, Paris, Seuil, 2006, p. 338339).

$57 \mathrm{La}$ question de l'appartenance est traitée dans Alain Badiou, Mathematics of the transcendental, London, Bloomsbury, 2014, p. 55 sq., et qui reprend le cœur des thèses défendues sur la vérité dans L'être et l'événement.

58 Alain Badiou, Court traité d'ontologie transitoire, Seuil, Paris, 1998, p. 198-200.

59 Alenka Zupančič, "The fifth condition », dans Think again: Badiou and the future of philosophy, dir. P. Hallward, London, Continuum, 2004, p. 191-201. 
L'essentiel est donc de parvenir encore à analyser ce mouvement d'institution d'un ordre co-constitué, stable, commun, sans qu'il reproduise une fois de plus l'illusion selon laquelle "l'identité est "posée" par l'institution comme un décret portant qu'il doit y avoir de l'identique 60 ». Suivant cette règle permanente de signification, la transmutation constante de modes d'existence par les savoir-faire techniques n'est que l'effet du faire social qui surpasse sa propre réification pour la transformer en image idéale de soi61. L'analyse de ce mouvement vers l'identique dans le moment toujours opérant de l'institution de l'être social-historique est d'autant plus importante que malgré les illusions qui s'y greffent, la question première n'est pas celle de la déconstruction ni d'ailleurs de la reconstruction. Ce qui s'y joue réside précisément dans l'entre-deux, non par négation «ni... ni », mais pas association « tout autant que ». Le propre du fantasme est de croire qu'il se saisit de l'image pour la posséder, comme si la part d'irréel et d'incertain dans ce qui est vécu pouvait être séparée d'une part vraie dont on parviendrait à produire le sens, sans le perdre. Or ce qui est en jeu dans le mouvement qui tend à suspendre l'indétermination pour y reconnaître l'institution possible d'une étape nouvelle vers l'identique, ce n'est pas l'un des termes du déplacement, mais l'oscillation entre ces termes, l'opération qui permet constamment de les combiner dans un mouvement de co-constitution comme ceux du voyant et $d u v^{62}$ : cette opération est la marque de l'incertitude du commun ou de sa non-identité première comme interruption possible de la répétition63.

Dans son beau commentaire de Paul64, Badiou revient sur cette question essentielle de l'interruption : celle-ci ne procède pas d'une négation (éliminer la différence propre à une occurrence), mais elle provient de la reconnaissance de l'indétermination qui est à la source de l'occurrence. C'est en fonction de cette indétermination que ce qui semble nous déterminer au plus haut point dans le réseau d'apparaissance du monde est éminemment réversible. « Il n’y a plus ni Juif ni Grec ; il n'y a plus ni esclave ni homme libre ; il n'y a plus ni homme ni femme... » (Ga 3, 28) L'égalité des occurrences n'apparaît comme option pertinente qu'à la condition d'éviter l'illusion d'une factualité absolue, c'est-à-dire prise imaginairement comme vérité définitive. Toute factualité peut être traversée et « resubjectivée », comme dit Badiou65. Elle ne fige pas l'identité à soi, mais renvoie au contraire à la non-identité

\footnotetext{
60 Cornelius Castoriadis, L'institution imaginaire de la société, op. cit., p. 306.

61 Ibid., p. 314.

62 Cornelius Castoriadis, Histoire et création, Textes philosophiques inédits (1945-1967), éd. Nicolas Poirier, Paris, Seuil, 2009, p. 166.

$63 C f$. Jacques Lenoble, "L'enjeu du dernier enseignement de Lacan : vers une approche réflexive du "Un réel” ", MIMESIS/Teoria e Critica della Regolazione Sociale, vol. 1, n 2, 2016, p. 11-40.

64 Alain Badiou, Saint Paul. La fondation de l'universalisme, Paris, PUF, 1997.

65 Ibid., p. 110.
} 
de l'indétermination qui permet l'interruption de la répétition dans le temps du faire social-historique. « Où Personne n'était, Nous devons devenir66»!

De ce point de vue, en restant attentif au geste psychanalytique, Castoriadis permet d'éviter l'idéalisation d'une norme d'apparaître fournie par le moment isolé d'une pragmatique interactionniste en sciences sociales. La fiction de l'espace logocentrique ou conversationnel impose un rapport à la normativité sociale des pratiques qu'elle ne remet plus en question, sous peine d'éliminer une compréhension positive du retour de l'indétermination par l'action anomique.

66 Cornelius Castoriadis, «Épilégomènes à une théorie de l'âme que l'on a pu présenter comme science », dans Les Carrefours du labyrinthe, I, Paris, Seuil, 1978, p. 80. 